\title{
Time-resolved SERS study of the oxygen reduction reaction in ionic liquid electrolytes for non-aqueous lithium- oxygen cells
}

\author{
Petar M. Radjenovic and Laurence J. Hardwick (iD *
}

Received 15th May 2017, Accepted 2nd June 2017

DOI: $10.1039 / c 7 f d 00170 c$

Superoxide $\left(\mathrm{O}_{2}{ }^{-}\right)$is the key intermediate formed during oxygen reduction in nonaqueous electrolytes. One significant obstacle towards the realisation of a practical lithium-oxygen $\left(\mathrm{Li}-\mathrm{O}_{2}\right)$ battery is electrolyte instability in the presence of radical oxides, principally superoxide. Here we use the Raman active bands of $\mathrm{O}_{2}{ }^{--}$as a diagnostic molecule for probing the influence of the electrolyte on reaction processes and intermediaries at the electrode surface. In situ surface enhanced Raman studies of the interface at a roughened Au electrode with controlled and dynamic surface potentials were performed in two ionic liquids with differing properties: 1-butyl-1-methylazepenium bis(trifluoromethanesulfonyl)imide (Aze $\left.{ }_{14} T F S I\right)$, which has a large/soft cation, and triethylsulfonium bis(trifluoromethanesulfonyl)imide (TESTFSI), which has a relatively small/hard and $\mathrm{e}^{-}$accepting cation. The counter-cation and potential were seen to significantly influence the radical nature, or Lewis basicity of $\mathrm{O}_{2}{ }^{-}$. The analysis of peak intensities and Stark shifts in $\mathrm{O}_{2}{ }^{-}$related spectral bands allowed for key information on its character and electrolyte interactions to be elucidated. Time-resolved studies of dynamic surface potentials permitted real time observation of the flux and reorientation of ions at the electrode/electrolyte interface.

\section{Introduction}

Superoxide, $\mathrm{O}_{2}{ }^{--}$, is the key reaction intermediary formed during the oxygen reduction reaction (ORR) at the cathode in lithium-oxygen $\left(\mathrm{Li}-\mathrm{O}_{2}\right)$ cells and can cause parasitic side reactions that limit the capacity and cycle life of the cell. Therefore, understanding the interaction of $\mathrm{O}_{2}{ }^{--}$with electrolyte provides valuable information for selecting or designing a more robust electrolyte.

The solvation properties of the electrolyte have been suggested to play an important role in the cathode intermediary reaction mechanism for some time. ${ }^{1}$ More recently, solvents used in the non-aqueous $\mathrm{Li}-\mathrm{O}_{2}$ electrolyte were 
shown to directly affect the ORR, inducing either a surface or solution reaction mechanism. ${ }^{2,3}$ The surface mechanism produces an insulating $\mathrm{Li}_{2} \mathrm{O}_{2}$ film that eventually builds up and passivates the cathode surface causing premature cell death. Whereas, the solution mechanism is favoured due to its ability to bypass passivation films forming surface agglomerates on the $\mathrm{O}_{2}$-cathode instead, extending the exposed cathode surface area for charge transfer until later into discharge, allowing for larger discharge capacities. ${ }^{2}$ Two distinct paths for inducing the solution mechanism have since emerged: [1] high Gutmann donor number (DN) and [2] high Gutmann acceptor number (AN) mechanisms. The Gutmann donor/acceptor numbers are empirically derived methods for estimating the affinity of a solvent for donating or accepting negative charge from solvated species and thus the strength of solvent-solute interactions. ${ }^{\mathbf{4}, 5}$

An ideal electrolyte should have an 'amphoteric-like' nature, with both a high DN and AN component capable of effectively stabilising and solvating both charged species, reducing their propensity to react together and increasing their solvation into the bulk electrolyte away from the cathode surface (provided the metal anode is adequately protected against dissolution reactions driven by $\mathrm{O}_{2}{ }^{\cdot-}$ (sol) crossover). Ionic liquids fit this model as they contain ions with both positive and negative formal charges. Many conventional organic solvents are unstable in the presence of lithium metal and/or reduced oxygen species. Even those electrolytes that have relatively good $\mathrm{Li}-\mathrm{O}_{2}$ electrochemistry (such as dimethyl sulfoxide, DMSO) have been shown to be unstable after prolonged exposure to these species, ${ }^{6-8}$ as well as in the presence of lithium metal, thus ruling them out as practical battery electrolytes. One method of overcoming this may be to use ionic liquids (ILs) as additives or neat ionic electrolytes. Ionic liquids possess a number of favourable properties for open 'air' batteries due to their innate conductivity, universally low vapour pressures, flammability and their high thermal, chemical and electrochemical stability..$^{9-17}$ Also, their properties can be tailored to application requirements (depending on constituent ions selected) making them extremely versatile solvent-electrolyte candidates. Schmeisser et al. ${ }^{9}$ correlated the relationship between the cation and anion of an IL with its acceptor and donor numbers. In general, increasing the mass and size or 'charge dissociation' across the cation/anion, decreases the AN/DN respectively of the IL. Larger, 'bulkier', heavier ions have slower kinetics, increased steric hindrance and are less able to interact with external charge. This can also be explained in terms of hard-soft acid-base (HSAB) theory. Larger, heavier ions are softer and interact less with hard counter ions; therefore, smaller IL cations will be expected to interact more with $\mathrm{O}_{2}{ }^{--}$than larger cations that have a high degree of charge dissociation. Considering the application of non-aqueous $\mathrm{Li}-\mathrm{O}_{2}$ cells, the evaluation of the chemical nature of $\mathrm{O}_{2}{ }^{--}$at the electrode interface provides valuable information for the ion selection process of an IL electrolyte. To probe this effect and the cations influence on $\mathrm{O}_{2}{ }^{--}$, a large charge dissociated cyclic alkylammonium cation, 1-butyl-1-methyl-azepenium $\left(\mathrm{Aze}_{14}{ }^{+}\right)$, and a relatively small cation, triethylsulfonium $\left(\mathrm{TES}^{+}\right)$, based ILs were selected for this work (Fig. 1). 


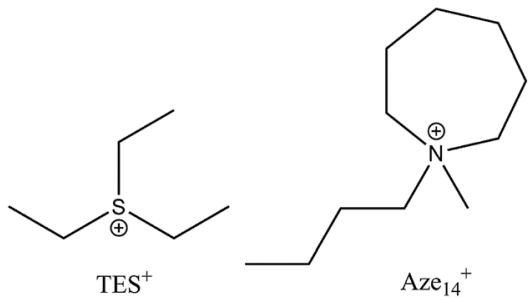

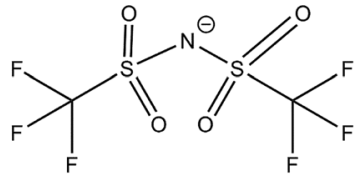

TFSI $^{-}$

Fig. 1 Chemical structures of ionic liquid cations and anion used in this work and the associated abbreviations for each constituent are presented.

\section{Experimental}

Electrochemical measurements were carried out within a glass, multi-necked vacuum tight cell within an inert atmosphere glovebox $\left(<30 \mathrm{ppm} \mathrm{O}_{2},<0.5 \mathrm{ppm}\right.$ $\mathrm{H}_{2} \mathrm{O}$ ) at ambient temperature. High purity oxygen $(\geq 99.999 \%)$ and argon $(\geq 99.998 \%$ ), dried by passing through multiple desiccant filled drying tubes, was used to degas and oxygenate electrolytes via Young tapped gas inlet and outlets. Polycrystalline gold $\mathrm{Au}$ ) working disc electrodes $(0.16 \mathrm{~cm}$ diameter $)$ were used as working electrodes. The electrode surfaces were polished mechanically with decreasing grain sized alumina slurries $(1.0 \mu \mathrm{m}, 0.3 \mu \mathrm{m}$ and $0.05 \mu \mathrm{m})$. These were washed in Milli-Q water $(18.2 \mathrm{M} \Omega)$ and sonicated before being dried at $120{ }^{\circ} \mathrm{C}$ under vacuum overnight. A flame annealed platinum coiled wire was used as a counter electrode and a silver wire as a quasi-reference electrode. The quasireference electrode was standardised against an internal ferrocene standard which has a potential of $+0.44 \mathrm{~V} v s$. NHE in each individual IL to determine the corresponding $\mathrm{Ag}^{+} / \mathrm{Ag}$ potential shift.

In situ SERS measurements were setup similarly using a multi-necked gas-tight glass cell fitted with a sapphire window (TMS Vacuum Components). The cell was specially designed to minimise the volume of ionic liquid required for each test $(<0.6 \mathrm{ml})$ (Fig. 2). Behind this window an electrochemically roughened Au working electrode was placed. The Au working electrode was cleaned (described previously) and roughened using an oxidation/reduction cycle (ORC) described by Tian et al. ${ }^{18}$ Spectra were recorded using a $50 \times$ objective on a Raman spectrometer (Renishaw Invia) with a $785 \mathrm{~nm}$ laser calibrated against a silicon wafer. SERS measurements of the surface were conducted by taking rapid ( 1.4 s) static spectra of the regions of interest. Due to the limited wavenumber range of the quick scans ( $c a .400 \mathrm{~cm}^{-1}$ region) spectra of $\nu_{\mathrm{O}-\mathrm{O}}$ and $\nu_{\mathrm{O}-\text { surf }}$ regions were taken on consecutive cyclic-voltammetry (CV) cycles. Comparing differences in spectra of the bulk IL and the surface at OCP with spectra of the surface at lower potentials shows the flux of species at the surface.

Aze $_{14}$ TFSI (provided by Queens University Belfast) ${ }^{19}$ and TESTFSI (Sigma, 99\% purity) were dried at $100-130{ }^{\circ} \mathrm{C}$ for between 1 and 2 days inside an in-house made vacuum tube in a sand bath using a heater stirrer (Assynt) under vacuum $(1 \times$ $10^{-6} \mathrm{~Pa}$ ) before being stored in an argon $(\geq 99.998 \%)$ atmosphere glovebox. Water content was reduced to 1-6 ppm for Aze ${ }_{14}$ TFSI and $<20 \mathrm{ppm}$ for TESTFSI, measured using a coulometric Karl Fischer titrator (Mettler-Toledo). TESTFSI is more hygroscopic and required longer drying times ( $\sim 3$ days). 


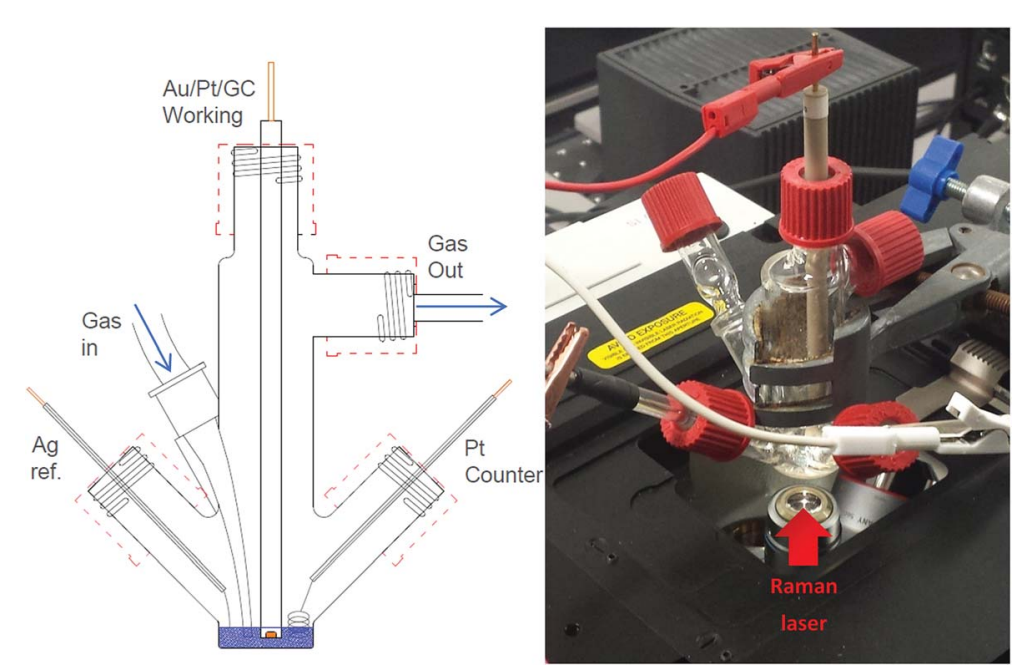

Fig. 2 Schematic of three-electrode, low-volume $(<0.6 \mathrm{ml})$, spectroelectrochemical cell (left) and photograph of cell setup over the microscope objective of the Raman spectrometer (right).

\section{Results and discussion}

As stated in the Experimental section Raman spectra were collected in rapid succession (every $\sim 1.4 \mathrm{~s}$ ) during potential cycling. The inherently short laser exposure times can lead to high background noise making enhancement active surfaces essential. However, with an optimum SERS electrode substrate, multiple spectra can be amassed with excellent signal-to-noise ratio allowing for detailed contour and multi-dimensional plots (Fig. 3) of bond vibrations to be generated showing the real-time flux of vibrational bonds at the surface.

In Fig. 3(a) and (c) the top part shows the variation of potential and current with respect to time during the CV scan. The corresponding contour plots (red colour being the most intense and purple the least) of Raman band intensities with respect to time are shown in the bottom part. The equivalent $3 \mathrm{D}$ plot of the data is shown in Fig. 3(b) and (d) with significant bands labelled on the plot. For both TESTFSI and Aze ${ }_{14}$ TFSI a number of potential dependent bands are observed to emerge and vanish as the potential is swept. As can be seen in the Fig. 3 plots, two spectral bands for $\mathrm{O}_{2}{ }^{--}$appear during ORR and disappear on the reverse scan: (1) the asymmetric dioxygen stretch, $\nu_{\mathrm{O}-\mathrm{O}},\left(c a .1100 \mathrm{~cm}^{-1}\right)$ and (2) the oxygensurface bond, $\nu_{\mathrm{O}-\text { surf }}\left(c a .470 \mathrm{~cm}^{-1}\right)$. The $\nu_{\mathrm{O}-\mathrm{O}}$ band position is heavily dependent on the immediate coordinating environment and counter-cation size. ${ }^{20}$ However, acidity/basicity of aqueous electrolytes, ${ }^{21,22}$ neighbouring anions in a doped structures ${ }^{23}$ and H-bonding impurities ${ }^{24}$ have also been shown to significantly influence the $\mathrm{O}_{2}{ }^{--}$bond force constant and $\nu_{\mathrm{O}-\mathrm{O}}$ spectral band position by directly interacting with $\mathrm{O}_{2}{ }^{\cdot-}$ charge. Additionally the counter-cation coordination strength, lattice parameters in superoxide salts or increasing mutual repulsion between electrolytic anions can also indirectly influence the $\mathrm{O}_{2}{ }^{--}$bond force constant causing shifts in $\nu_{\mathrm{O}-\mathrm{O}}$. 

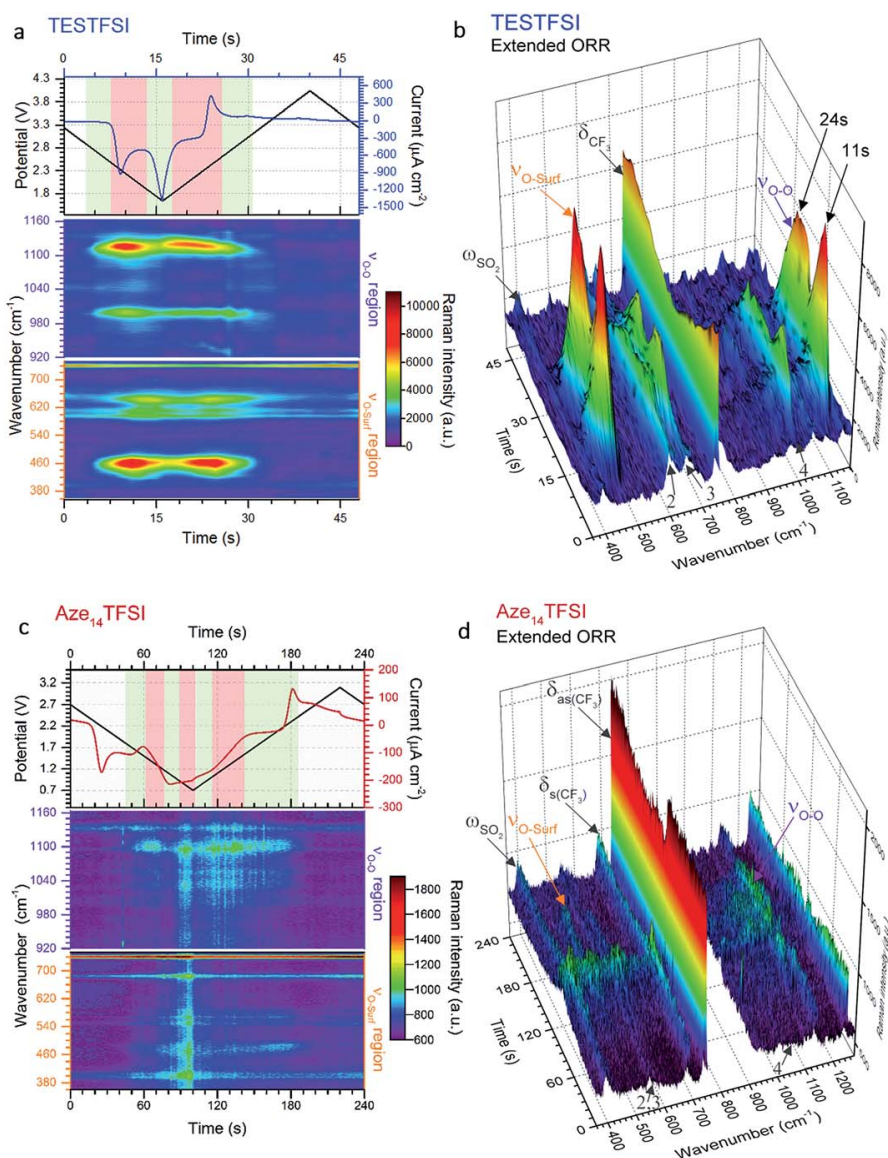

Fig. 3 Dynamic surface potential in situ SERS multi-dimensional plots. (a) and (c): Top: potential (black line)/current (blue line) vs. time plots of ORR/OER in oxygenated TESTFSI analogous with CV in Fig. $4(\mathrm{~b})$ and (d) and contour plots of SERS data in $\nu_{\mathrm{O}-\mathrm{O}}$ and $\nu_{\mathrm{O}-\text { surf }}$ regions respectively at corresponding times with the electrochemistry (analogous with SERS spectra in Fig. 4(a) and (c)). Regions where key surface species and $\mathrm{O}_{2}{ }^{\cdot-}$ vibrational intensities are present and strongest/weakest highlighted in red/green respectively (top). Regions of low and high $\nu_{\mathrm{O}-\mathrm{O}}$ and $\nu_{\mathrm{O}-\text { surf }}$ band intensities highlighted in green and red in the electrochemical plots. (b) and (d): 3D visual plot of spectral changes with time. Changes in intensity of $\delta \mathrm{CF}_{3}$ bond are more clearly visible than in the contour plot. These are associated with reduced anion present at the surface at lower potentials that recovers when potentials are returned to positive values and anions repopulate the double layer. Decreasing $\mathrm{O}_{2}{ }^{--}$and electrolyte band (peak 2-4) intensities at low potentials are also clearly visible.

Fig. 4(a) and (c) show the corresponding selected stacked spectra of the data shown in Fig. 3. From this plot band shifts of $\nu_{\mathrm{O}-\mathrm{O}}$ and $\nu_{\mathrm{O}-\text { surf }}$ become more apparent. The top half of Fig. 4(b) and (d) show ORR/OER cyclic voltammograms (CVs) of oxygen saturated TESTFSI (relatively hard cation) and Aze ${ }_{14}$ TFSI (soft cation) ILs respectively during standard (lower potential limit of $2.0 \mathrm{~V}$ ) and extended (lower potential limit down to either 1.6 or $0.6 \mathrm{~V}$ ) potential cycling. Normal reduction cycles are shown by dashed lines, however, in order to improve 
a

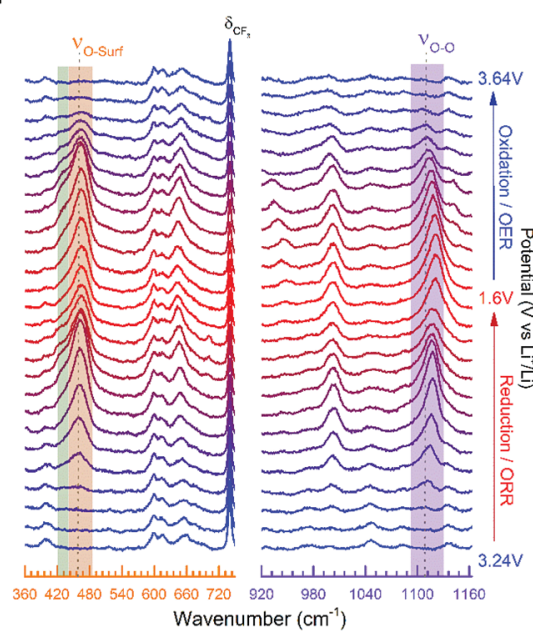

b TESTFSI Potential $\left(\vee v s\right.$ Li $\left.i^{+} / \mathrm{Li}\right)$

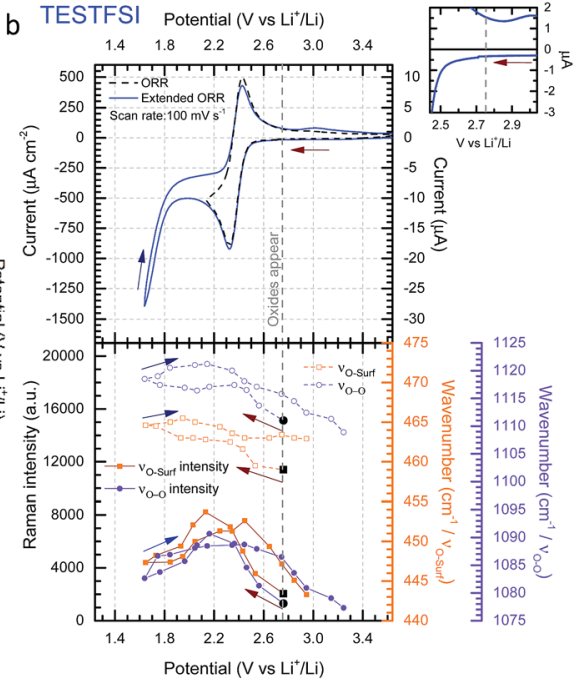

d

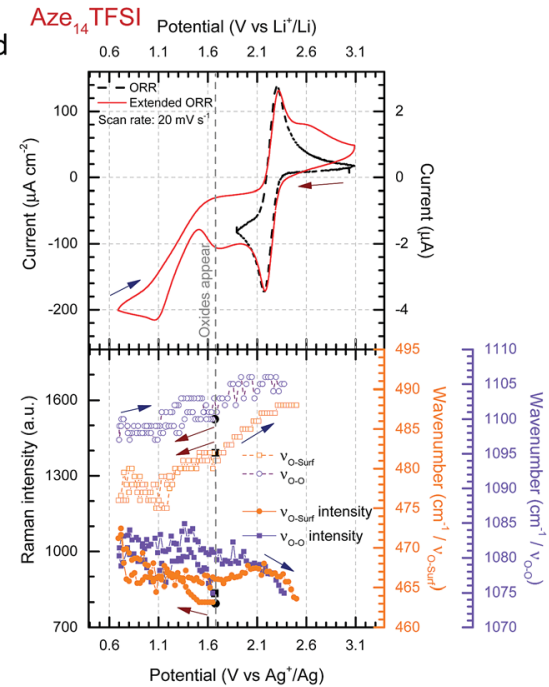

Fig. 4 Dynamic surface potential in situ SERS. (a) and (c) Selection of stacked SERS spectra of the rAu working electrode surface collected in rapid succession in oxygen saturated TESTFSI and $\mathrm{Aze}_{14}$ TFSI respectively. Left and right are scans of $\nu_{\mathrm{O}-\text { surf }}$ and $\nu_{\mathrm{O}-\mathrm{O}}$ wavenumber regions respectively. (b) Top, CVs of oxygenated TESTFSI at $100 \mathrm{mV} \mathrm{s}^{-1}$ with normal ORR/OER cycle (dashed line) and extended reduction cycle (blue line). Top right inset shows potential region where $\mathrm{O}_{2}{ }^{\cdot-}$ bands first appear with small current response due to an initial adsorbed dioxygen surface layer prior to ORR. Bottom, $\nu_{\mathrm{O}-\mathrm{O}}$ and $\nu_{\mathrm{O}-\text { surf }}$ wavenumber position and intensity plots vs. potential. There is a visible blue-Stark shift. (d) Top, CVs of oxygenated Aze ${ }_{14}$ TFSI with normal ORR/OER cycle (black dashed line) and extended reduction cycle (red line). Bottom, $\nu_{\mathrm{O}-\mathrm{O}}$ and $\nu_{\mathrm{O}-\text { surf }}$ wavenumber position and intensity plots versus potential. There is a visible red-Stark shift. Both (b) and (d): starting data points when $\mathrm{O}_{2}{ }^{-}$bands appear shown in black, where possible coloured arrows are included as visual aids indicating reduction (red) and oxidation (blue) directions of potential scanning. 
visibility and investigate the influence of potential on the $\mathrm{O}_{2}{ }^{--}$bond vibrations, spectra were collected during reduction cycles where the potential was taken to the second reduction maxima generally associated with $\mathrm{O}_{2}{ }^{2-}$ production. ${ }^{25}$ It should be noted within this study no bands pertaining to $\mathrm{O}_{2}{ }^{2-}$ were observed. During full cycles, rapid spectra of the roughened gold $(\mathrm{rAu})$ working electrode surface were continuously collected. The appearance and disappearance of characteristic $\nu_{\mathrm{O}-\mathrm{O}}$ and $\nu_{\mathrm{O}-\text { surf }}$ spectral bands (highlighted in purple and orange respectively) were apparent in both ILs as the potential was swept. However, the signals for $\mathrm{O}_{2}{ }^{-}$bands were almost an order of magnitude weaker in the $\mathrm{Aze}_{14}$ TFSI than in TESTFSI, and were only detected at potentials after the reduction maxima and slower scan rates were required $\left(20 \mathrm{mV} \mathrm{s}^{-1}\right.$ in Aze $_{14}$ TFSI compared with $100 \mathrm{mV} \mathrm{s}^{-1}$ in TESTFSI). Similarly weak $\mathrm{O}_{2}{ }^{\cdot-}$ bands have been reported previously with alkylammonium salts dissolved in $\mathrm{MeCN}$ where the $\nu_{\mathrm{O}-\mathrm{O}}$ signal was much stronger in smaller tetraethylammonium than tetrabutylammonium based electrolytes. ${ }^{20}$ Both bands were observed to blue or red Stark shift with decreasing potential in TESTFSI and Aze ${ }_{14}$ TFSI respectively, and a plot of their peak fitted band positions and intensities are shown in the bottom half of Fig. 4(b) and (d). Electrolyte peaks labelled 2-4, were also observed to be potential dependant in both TESTFSI and Aze ${ }_{14}$ TFSI. Identity of key potential dependent peaks of both ionic liquids, with observations are summarised within Table 1.

Variances in spectral band intensities give qualitative information on surface coverage, molecular orientation and the concentration of species at the electrode/ electrolyte interface during potential cycling. Differences (or shifts) in $\nu_{\mathrm{O}-\mathrm{O}}$ band positions between electrolytes from lower (red) to higher (blue) wavenumbers generally indicate more ionic or covalent, respectively, interactions between $\mathrm{O}_{2}{ }^{\cdot-}$ and its coordinating environment. A more ionic $\mathrm{O}_{2}{ }^{--}$species is a less coordinated, more radical, harder Lewis base whilst a more covalent $\mathrm{O}_{2}{ }^{-}-$species is a more coordinated, softer Lewis base. Likewise, blue or red Stark shifts in $\nu_{\mathrm{O}-\mathrm{O}}$ bands during potential cycling indicate surface potential induced fluctuations in the ionic/covalent character, respectively, of generated $\mathrm{O}_{2}{ }^{--}$. The Stark effect, being consistent with Guoy-Chapman theory, is heavily dependent on the distance of the probe molecule $\left(\mathrm{O}_{2}{ }^{-}\right)$from the surface and therefore indicates observation of species bonded, adsorbed or adjacent to the surfaces. ${ }^{26}$ For $\nu_{\mathrm{O}-\text { surf }}$, differences in band positions between electrolytes and red/blue Stark shifts indicate longer (weaker) and shorter (stronger) adsorption bonds, respectively. Both $\nu_{\mathrm{O}-\mathrm{O}}$ and $\nu_{\mathrm{O}-}$ surf bands are closely related appearing on ORR and disappearing on OER; though there were significant differences between the two IL electrolytes which will be discussed. As such, the focus on the effect of the cation on electrochemically generated surface $\mathrm{O}_{2}{ }^{--}$by varying the substituent cation in two distinct ILs with either a relatively small, TES, or large charge dissociated cyclic alkylammonium, $\mathrm{Aze}_{14}$, as the cation is given thorough consideration.

In TESTFSI the $\mathrm{O}_{2}{ }^{\cdot-}$ bond vibrations first appear (weakly) at $2.75 \mathrm{~V} v s . \mathrm{Li}^{+} / \mathrm{Li}$ in TESTFSI and grow in intensities to a maximum just after the reduction potential current maxima. The top right inset of Fig. 4(b) shows this to be the precise onset point of an exponential decrease in current and beginning of bulk $\mathrm{O}_{2}$ reduction. This is below the thermodynamic reduction potential of oxygen reduction in lithiated systems $\left(2.97 \mathrm{~V} v \text { s. } \mathrm{Li}^{+} / \mathrm{Li}\right)^{27}$ but significantly higher than the $E_{1 / 2}$ of the ORR/OER reaction in TESTFSI. 
Table 1 Summary of key ionic liquid bands. $\mathrm{C}^{+}$refers to cation, either TES ${ }^{+}$or $\mathrm{Aze}_{14}{ }^{+}$(note peak 1 is the designation of the flat conformer of adsorbed superoxide at ca. $430 \mathrm{~cm}^{-1}$ )

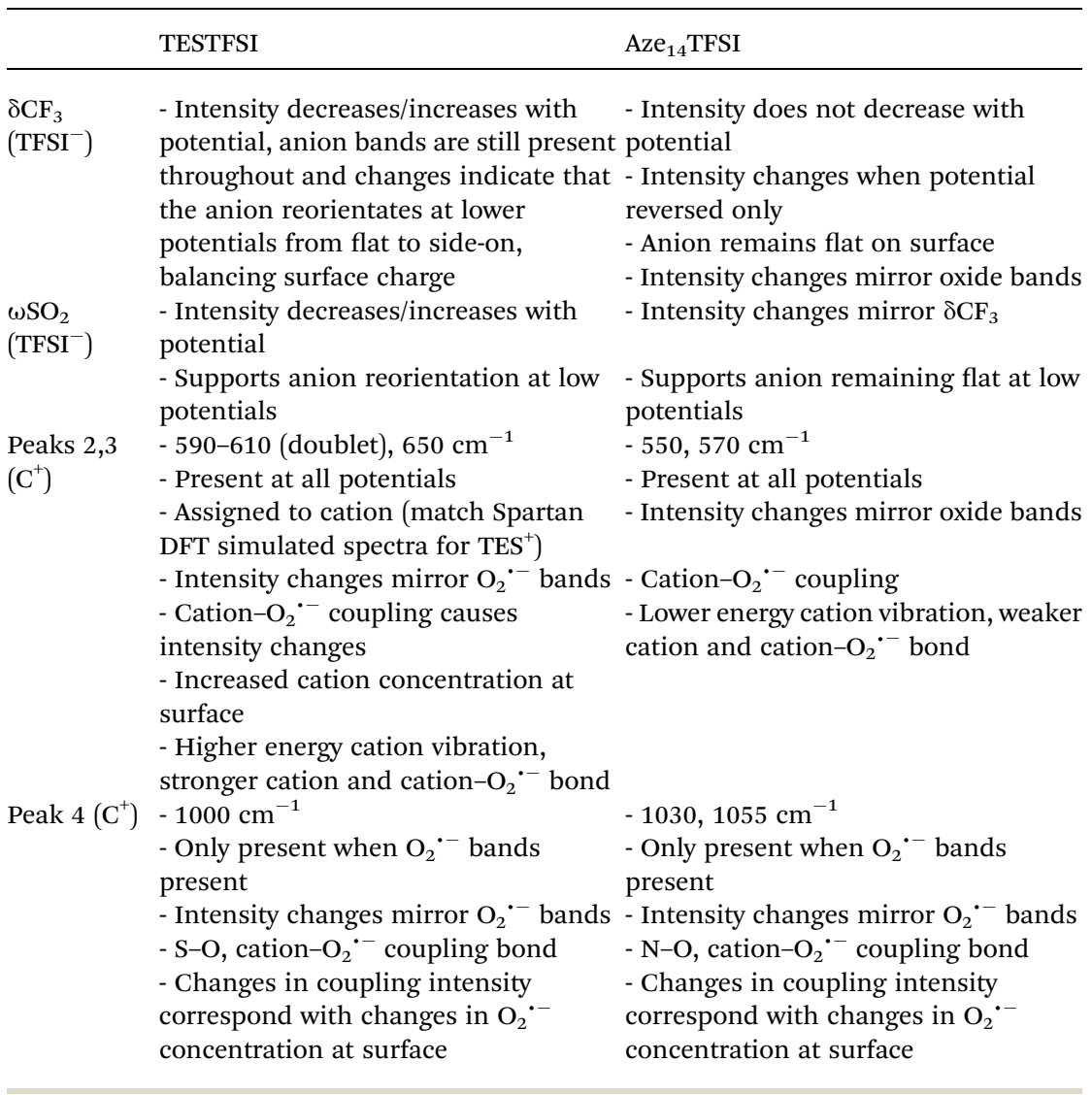

Comparing the $\mathrm{O}_{2}{ }^{--}$bands between the two ILs the $\nu_{\mathrm{O}-\mathrm{O}}$ band is much higher wavenumber for TESTFSI $\left(1120 \mathrm{~cm}^{-1}\right)$ than in the Aze ${ }_{14}$ TFSI $\left(1105 \mathrm{~cm}^{-1}\right)$. This agrees with the trend in coordinating cation size observed in our previous work on ORR within tetraalkylammonium based electrolytes, ${ }^{20}$ indicating a more Lewis basic " $\mathrm{O}_{2}{ }^{--}$" is generated at the surface in Aze ${ }_{14}$ TFSI than TESTFSI, due to being less strongly coordinated by the $\mathrm{Aze}_{14}{ }^{+}$counter cation in the electrolyte. There is a similar difference in the $\nu_{\mathrm{O}-\text { surf }}$ bands, with lower wavenumber values in TESTFSI $\left(466 \mathrm{~cm}^{-1}\right)$ than Aze ${ }_{14}$ TFSI $\left(488 \mathrm{~cm}^{-1}\right)$. Lower $\nu_{\mathrm{O}-\text { surf }}$ wavenumbers indicate longer, weaker oxygen-to-surface adsorption bonds that suggests that some $\mathrm{e}^{-}$density is shared with the electrode surface.

Dioxygen ligand-to-metal bonds in organometallic chemistry are analogous to surface adsorption bonds and can help rationalise the bond structures of the observed adsorption bond vibrations. The three most common dioxygen-metal bond structures are side-on (flat), kinked and bent, shown in Fig. 5. The longest/ weakest adsorption bond for the side-on, or flat, dioxygen-metal structure is often associated with the peroxo-complex ${ }^{28,29}$ though side-on superoxo-complexes have also been reported..$^{30,31}$ The energy of the dioxygen $\pi^{*}$ levels lies below that of metal d-orbitals therefore the flat structure is mainly composed of d-to- $\pi^{*}$ 
bonding with equal bonding between both oxygen atoms and the metal centre, ${ }^{28}$ provided that the metal Fermi level is in a state that permits it. The superoxoligand however is known to be preferentially bent-bonded to metal sites, through ligand-to-metal $\sigma$ donation. Back donation from the metal into the antibonding orbitals can occur, if the surface energetics permit, which weakens the O-to-surface bond, relative to the bent-bonded structure, lowering the bond force constant producing a longer kinked adsorption bond. ${ }^{28} \mathrm{~A}$ kinked bond has characteristics between the flat and bent bonded species with both oxygen atoms interacting to differing degrees with the metal centre and at an angle with the surface, the extent of back bonding and coordination dictates the angle.

Aze $_{14}$ TFSI is comprised of soft poorly accepting and donating ions. The weak accepting nature of the electrolyte increases the propensity of adsorbed $\mathrm{O}_{2}{ }^{--}$to share charge with the surface rather than the electrolyte. Giving rise to higher $\nu_{\mathrm{O}-}$ surf wavenumbers indicative of more energetic shorter bond vibrations in $\mathrm{Aze}_{14 \text { - }}$ TFSI relative to TESTFSI. This $\nu_{\mathrm{O}-\text { surf }}$ vibration can be attributed to a bent bonded structure that has little or no surface back bonding.

For $\mathrm{Aze}_{14}$ TFSI, both $\mathrm{O}_{2}{ }^{--}$bands were seen to red-Stark shift with decreasing surface potentials. Shifting the electrode potential negative increases the Fermi level of surface molecules, inducing coulombic repulsion between the surface and adsorbed anions and molecules with negative dipoles. When the electrolyte has a poor accepting nature that is less effective at balancing excess negative surface charge, as in Aze $_{14}$ TFSI, then surface $\mathrm{e}^{-}$density will be transferred and concentrated in any free molecular orbitals of surface bonded or adsorbed species closest in energy. In the case of $\mathrm{O}_{2}{ }^{--}$this will be the $\pi^{*}$ orbital, therefore back bonding increases with decreasing potentials and the bond becomes longer and increasingly kinked. This can also be explained as the increasingly negative surface charge contributes more $\mathrm{e}^{-}$density to surface adsorbed species reducing the effective nuclear charge experienced by valence bond electrons, causing both the $\nu_{\mathrm{O}-\text { surf }}$ and dioxygen bonds to weaken and lengthen. Hence a concomitant redStark shift in the $\nu_{\mathrm{O}-\mathrm{O}}$ bond vibrations of $\mathrm{O}_{2}{ }^{--}$close to the surface. This charge localisation on $\mathrm{O}_{2}{ }^{--}$at low potentials is indicative of a more Lewis basic species being generated at the surface with a weaker adsorption bond.

For TESTFSI, which can be considered as an $\mathrm{O}_{2}{ }^{--}$accepting electrolyte, $\nu_{\mathrm{O}-\text { surf }}$ is significantly lower than Aze $_{14}$ TFSI $\left(\sim 16-27 \mathrm{~cm}^{-1}\right)$, see Table 2, indicating a propensity for valence $\mathrm{e}^{-}$density on $\mathrm{O}_{2}{ }^{\cdot-}$ to interact more with the electrolyte

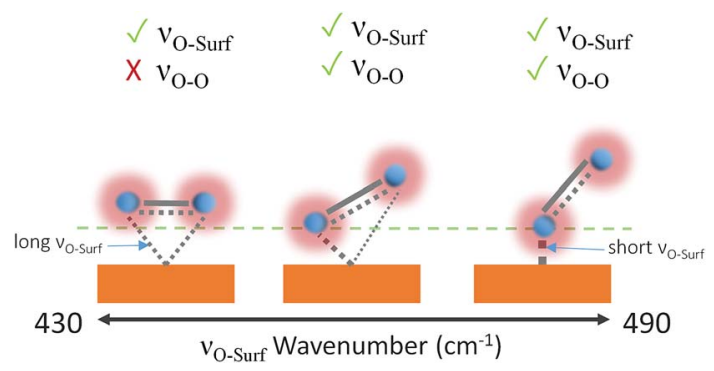

Fig. 5 Metal-to-dioxygen surface bond structures left to right: flat, kinked and bent. Visible bond vibrations based on the surface selection rule are shown above each structure. ${ }^{28,29,32}$ The tick and cross indicate which bands will be observable by Raman. 
than the surface indicating a weak adsorption bond with a kinked structure and some surface back bonding. An additional shoulder at $\sim 430 \mathrm{~cm}^{-1}$, labelled peak 1 (area highlighted in green in Fig. 4(a)), is also present at ORR/OER surface potential above $1.8 \mathrm{~V} v s$. $\mathrm{Li}^{+} / \mathrm{Li}$. Peak 1 has been characterised previously as a conformationally flat $\mathrm{O}_{2}{ }^{-}$- surface species on catalytically active $\mathrm{Pt}$ and $\mathrm{Pd}$ surfaces using SHINERS. ${ }^{33}$ Raman selection rules dictate that bond vibrations that are perpendicular to the surface are not visible. $\nu_{\mathrm{O}-\mathrm{O}}$ would therefore be absent or weak when a conformationally flat species is prominent at the surface.

This was the case when holding the potential at onset reduction potentials (Fig. 6), however at the same potential during cycling only a mixed (predominantly kinked) species is present. Therefore, the kinked structure appears to be kinetically more stable during potential cycling when the double layer is in flux; yet when the potential is held and the double layer equilibrates, the $\mathrm{O}_{2}{ }^{\cdot-}$ relaxes to a conformationally flat species. This was visible at onset potentials in TESTFSI electrolyte, but not in $\mathrm{Aze}_{14}$ TFSI. However, this effect was also present and far more pronounced in other ionic liquid electrolytes evaluated, not shown here. At extremely low potentials, below $1.8 \mathrm{~V} v s . \mathrm{Li}^{+} / \mathrm{Li}$, peak 1 disappears completely with only the $\nu_{\mathrm{O}-\mathrm{O}}$ (kinked) species visible. Blue-Stark shifts of 4 and $7 \mathrm{~cm}^{-1}$ were present with decreasing potentials. These observations suggest that the flat surface bond becomes less kinked and back bonding with the surface decreases with decreasing potential, the opposite of what was observed in Aze $_{14}$ TFSI. Counterintuitively, this would suggest that the $\pi^{*}$ orbital is being depleted of charge as the surface becomes increasingly more negative. This can be accounted for by considering changes within the double layer. When the surface potential is decreased the ionic liquid double layer becomes increasingly populated with positively charged species ${ }^{34}$ where the anions initially reorientate, followed by their expulsion ${ }^{35}$ in order to balance surface charge.

This was apparent in TESTFSI (Fig. 3(a) and (b)) by a continuous decrease in the intensity of the characteristic $\delta \mathrm{CF}_{3}$ vibration of the TFSI anion $\left(740 \mathrm{~cm}^{-1}\right)^{36}$ with lowering of potential. The $\delta \mathrm{CF}_{3}$ intensity increased again when the direction of scanning was reversed due to the anion repopulating the double layer. Furthermore, as the potential decreases a broad $\mathrm{Au}-\mathrm{S}$ adsorption band from the $\mathrm{S}$ in TES (200-300 $\mathrm{cm}^{-1}$ ) appears with ORR, indicative of increased cation population in the double layer (Fig. 6). Bands in the range of $600-700 \mathrm{~cm}^{-1}$ are seen to change in shape and intensity with respect to potential, which may arise from conformational changes of TES at the electrode surface, as seen previously for tetraalklyammonium cations. ${ }^{20}$ In addition, the intensity of electrolyte related peaks 2-4 of TESTFSI mirrored the intensity changes in the $\nu_{\mathrm{O}-\text { surf }}$ bond vibration

Table 2 Summary of $\nu_{\mathrm{O}-\mathrm{O}}$ and $\nu_{\mathrm{O} \text {-surf }}$ bond vibrations $\left(\nu_{x}\right)$ at the $\mathrm{rAu}$ interface in oxygen saturated TESTFSI and Aze 14 TFSI. $\Delta \nu_{x}$ denotes difference between highest and lowest values observed from Stark shifts

\begin{tabular}{lllr}
\hline & & $\nu_{x} / \mathrm{cm}^{-1}$ & $\Delta \nu_{x} / \mathrm{cm}^{-1}$ \\
\hline \multirow{2}{*}{ TESTFSI } & $\nu_{\mathrm{O}-\text { surf }}$ & $459-466$ & 7 \\
& $\nu_{\mathrm{O}-\mathrm{O}}$ & $1109-1120$ & 11 \\
Aze $_{14}$ TFSI & $\nu_{\mathrm{O}-\text { surf }}$ & $475-488$ & 13 \\
& $\nu_{\mathrm{O}-\mathrm{O}}$ & $1097-1105$ & 8
\end{tabular}




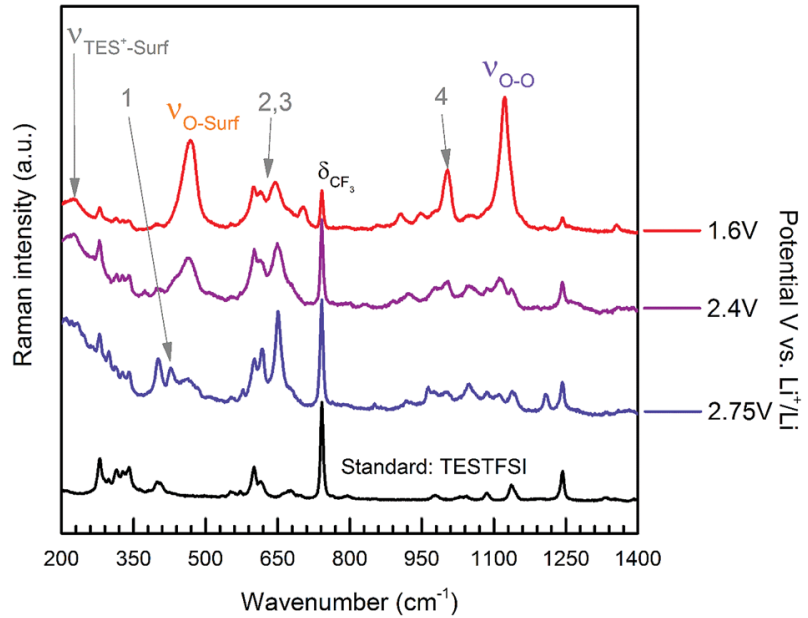

Fig. 6 Extended spectra acquired with surface potential control at specified held potentials of ORR in oxygen saturated TESTFSI.

(Fig. 3(a) and (b)). Peak $5\left(\sim 1001 \mathrm{~cm}^{-1}\right)$ similarly mirrored the $\nu_{\mathrm{O}-\mathrm{O}}$ bond vibration, however it was dependant on the ORR/OER potentials appearing and disappearing in tandem with $\nu_{\mathrm{O}-\mathrm{O}}$. Thereby showing a strong correlation of population of interfacial species from the electrolyte with respect to $\mathrm{O}_{2}{ }^{\cdot-}$. Similar electrolyte conformational changes were present, but much more difficult to observe in Aze $_{14}$ TFSI as shown in the contour plots in Fig. 3(c) and (d) due to the greater signal-to-noise ratio.

\section{Conclusions}

The cation effect on the radical character of $\mathrm{O}_{2}{ }^{--}$in non-aqueous ionic liquid media was investigated with surface enhanced Raman spectroscopy. Two ionic liquids with differing properties: 1-butyl-1-methyl-azepenium bis(trifluoromethanesulfonyl)imide (Aze ${ }_{14} \mathrm{TFSI}$ ), which has a large/soft cation, and triethylsulfonium bis(trifluoromethanesulfonyl)imide (TESTFSI), which has a relatively small/hard and $\mathrm{e}^{-}$accepting cation were investigated.

Substantial surface enhancements to the Raman signal permitted the accumulation of a Raman spectrum within $c a$. 1.4 seconds with sufficient signal-tonoise ratio. This allowed the observation of the effect of dynamic potential sweeping on both the appearance/disappearance and influence of $\mathrm{O}_{2}{ }^{\cdot-}$ upon the anion and cations of the ionic liquids to be evaluated.

The experiential trends provide insight into the nature of $\mathrm{O}_{2}{ }^{--}$and its character in non-aqueous purely ionic media. By contrasting the surface adsorbed $\mathrm{O}_{2}{ }^{\cdot-}$ bands between the two ILs the $\nu_{\mathrm{O}-\mathrm{O}}$ bands were found to occur at a much higher wavenumber for TESTFSI $\left(1120 \mathrm{~cm}^{-1}\right)$ than in the Aze ${ }_{14}$ TFSI $\left(1105 \mathrm{~cm}^{-1}\right)$ and indicates that a more Lewis basic superoxide is generated at the gold electrode surface in Aze $_{14}$ TFSI than TESTFSI due to being less strongly coordinated by the Aze $_{14}$ counter cation in the electrolyte. There was a similar difference in the $\nu_{\mathrm{O}-\text { surf }}$ bands with lower values in TESTFSI than Aze ${ }_{14}$ TFSI $\left(466 \mathrm{~cm}^{-1}\right.$ vs. 488 
$\mathrm{cm}^{-1}$ ). Lower $\nu_{\mathrm{O} \text {-surf }}$ wavenumbers indicate a longer, weaker oxygen-to-surface adsorption bond.

The differing effect upon the radical nature (Lewis basicity) of $\mathrm{O}_{2}{ }^{\cdot-}$ at the interface depending on choice of ionic liquid opens up a promising avenue of research, given the chemical flexibility of ionic liquids to be able to 'design' an optimum electrolyte system that provides a stable ionic medium to permit longlife and reversible cycling of $\mathrm{Li}-\mathrm{O}_{2}$ cells. If such an electrolyte can then also be tailored to provide acceptable levels of oxygen solubility and diffusivity, then progress towards the realisation of practical $\mathrm{Li}-\mathrm{O}_{2}$ cells becomes closer. Undoubtedly our observations on the cation effect on the nature of $\mathrm{O}_{2}{ }^{\cdot-}$ will need to be verified with further SERS studies on additional ionic liquids of differing cations and anions, and this will be the subject of future investigations.

\section{Acknowledgements}

We recognise funding from the joint Engineering and Physical Sciences Research Council (EPSRC) and Innovate UK grant (Practical Lithium-Air Batteries) EP/ L505274/1 that enabled this work. Dr Peter Goodrich and Professor Christopher Hardacre at Queens University Belfast for the supply of ionic liquids, as well scientific discussions with Dr Sarah Ball, at Johnson Matthey, are gratefully acknowledged.

\section{References}

1 C. O. Laoire, S. Mukerjee, E. J. Plichta, M. A. Hendrickson and K. M. Abraham, Rechargeable Lithium/TEGDME-LiPF $6 / \mathrm{O}_{2}$ Battery, J. Electrochem. Soc., 2011, 158, A302.

2 L. Johnson, et al., The role of $\mathrm{LiO}_{2}$ solubility in $\mathrm{O}_{2}$ reduction in aprotic solvents and its consequences for $\mathrm{Li}-\mathrm{O}_{2}$ batteries, Nat. Chem., 2014, 6, 1091-1099.

3 N. B. Aetukuri, et al., Solvating additives drive solution-mediated electrochemistry and enhance toroid growth in non-aqueous $\mathrm{Li}-\mathrm{O}_{2}$ batteries, Nat. Chem., 2014, 7, 50-56.

4 V. Gutmann, Solvent effects on the reactivities of organometallic compounds, Coord. Chem. Rev., 1976, 18, 225-255.

5 B. M. Gallant, et al., Influence of $\mathrm{Li}_{2} \mathrm{O}_{2}$ morphology on oxygen reduction and evolution kinetics in $\mathrm{Li}-\mathrm{O}_{2}$ batteries, Energy Environ. Sci., 2013, 6, 2518.

6 D. G. Kwabi, et al., Chemical Instability of Dimethyl Sulfoxide in Lithium-Air Batteries, J. Phys. Chem. Lett., 2014, 5, 2850-2856.

7 D. Sharon, et al., Oxidation of Dimethyl Sulfoxide Solutions by Electrochemical Reduction of Oxygen, J. Phys. Chem. Lett., 2013, 4, 3115-3119.

8 D. Sharon, et al., On the Challenge of Electrolyte Solutions for Li-Air Batteries: Monitoring Oxygen Reduction and Related Reactions in Polyether Solutions by Spectroscopy and EQCM, J. Phys. Chem. Lett., 2013, 4, 127-131.

9 M. Schmeisser, P. Illner, R. Puchta, A. Zahl and R. van Eldik, Gutmann donor and acceptor numbers for ionic liquids, Chem. - Eur. J., 2012, 18, 10969-10982.

10 A. Kokorin, Ionic Liquids: Theory, Properties, New Approaches, InTech, 2011.

11 O. U. Ahmed, F. S. Mjalli, T. Al-Wahaibi, Y. Al-Wahaibi and I. M. AlNashef, Stability of Superoxide Ion in Phosphonium-Based Ionic Liquids, Ind. Eng. Chem. Res., 2015, 54, 2074-2080. 
$12 \mathrm{~S}$. Das, et al., Instability of Ionic Liquid-Based Electrolytes in $\mathrm{Li}-\mathrm{O}_{2}$ Batteries, $J$. Phys. Chem. C, 2015, 119, 18084-18090.

13 D. R. MacFarlane, et al., Energy applications of ionic liquids, Energy Environ. Sci., 2014, 7, 232.

14 T. Zhang and Z. Wen, A High-Rate Ionic Liquid Lithium- $\mathrm{O}_{2}$ Battery with LiOH Product, J. Phys. Chem. C, 2017, 121, 5968-5973.

15 N. V. Plechkova and K. R. Seddon, Applications of ionic liquids in the chemical industry, Chem. Soc. Rev., 2008, 37, 123-150.

16 D. R. MacFarlane, et al., Ionic liquids and their solid-state analogues as materials for energy generation and storage, Nat. Rev. Mater., 2016, 1, 15005.

17 A. R. Neale, et al., Effect of cation structure on the oxygen solubility and diffusivity in a range of bis\{(trifluoromethyl)sulfonyl $\}$ imide anion based ionic liquids for lithium-air battery electrolytes, Phys. Chem. Chem. Phys., 2016, 18, 11251-11262.

18 B. Ren, X.-B. Lian, J.-F. Li, P.-P. Fang, Q.-P. Lai and Z.-Q. Tian, Spectroelectrochemical flow cell with temperature control for investigation of electrocatalytic systems with surface-enhanced Raman spectroscopy, Faraday Discuss., 2009, 140, 155-165.

19 S. Pohlmann, et al., Azepanium-based ionic liquids as green electrolytes for high voltage supercapacitors, J. Power Sources, 2015, 273, 931-936.

20 I. M. Aldous and L. J. Hardwick, Influence of tetraalkylammonium cation chain length on gold and glassy carbon electrode interfaces for alkali metaloxygen batteries, J. Phys. Chem. Lett., 2014, 5, 3924-3930.

$21 \mathrm{X}$. Li and A. Gewirth, Oxygen electroreduction through a superoxide intermediate on Bi-modified Au surfaces, J. Am. Chem. Soc., 2005, 127, 52525260.

$22 \mathrm{~J}$. Kim and A. Gewirth, Mechanism of Oxygen Electroreduction on Gold Surfaces in Basic Media, J. Phys. Chem. B, 2006, 110, 2565-2571.

23 W. Holzer, W. F. Murphy, H. J. Bernstein and J. Rolfe, Raman spectrum of $\mathrm{O}_{2}{ }^{-}$ ion in alkali halide crystals, J. Mol. Spectrosc., 1968, 26, 543-545.

24 P. D. C. Dietzel, R. K. Kremer and M. Jansen, Tetraorganylammonium Superoxide Compounds: Close to Unperturbed Superoxide Ions in the Solid State, J. Am. Chem. Soc., 2004, 126, 4689-4696.

25 A. W. Lodge, M. J. Lacey, M. Fitt, N. Garcia-Araez and J. R. Owen, Critical appraisal on the role of catalysts for the oxygen reduction reaction in lithium-oxygen batteries, Electrochim. Acta, 2014, 140, 168-173.

26 V. Oklejas, C. Sjostrom and J. M. Harris, Surface-Enhanced Raman Scattering Based Vibrational Stark Effect as a Spatial Probe of Interfacial Electric Fields in the Diffuse Double Layer, J. Phys. Chem. B, 2003, 107, 7788-7794.

27 Y.-C. Lu, et al., Platinum-gold nanoparticles: a highly active bifunctional electrocatalyst for rechargeable lithium-air batteries, J. Am. Chem. Soc., 2010, 132, 12170-12171.

28 M. R. Albert and J. T. Yates, The surface scientist's guide to organometallic chemistry, American Chemical Society, 1987.

29 L. Vaska, Dioxygen-metal complexes: toward a unified view, Acc. Chem. Res., 1976, 9, 175-183.

$30 \mathrm{C}$. $\mathrm{Cu}$, et al., A Monomeric Side-On Superoxocopper(II) Complex: $\mathrm{Cu}(\mathrm{Oz})(\mathrm{HB}(3-$ tBu-5-iPrpz) $)_{3}$ ), J. Am. Chem. Soc., 1994, 12, 12079-12080. 
31 J. W. Egan, B. S. Haggerty, A. L. Rheingold, S. C. Sendlinger and K. H. Theopold, Crystal Structure of a Side-On Superoxo Complex of Cobalt and Hydrogen Abstraction by a Reactive Terminal Oxo Ligand, J. Am. Chem. Soc., 1990, 112, 2445-2446.

32 C. H. Barlow, J. C. Maxwell, W. J. Wallace and W. S. Caughey, Elucidation of the mode of binding of oxygen to iron in oxyhemoglobin by infrared spectroscopy, Biochem. Biophys. Res. Commun., 1973, 55, 91-95.

33 T. A. Galloway and L. J. Hardwick, Utilizing in situ Electrochemical SHINERS for Oxygen Reduction Reaction Studies in Aprotic Electrolytes, J. Phys. Chem. Lett., 2016, 7, 2119-2124.

34 K. Motobayashi, K. Minami, N. Nishi, T. Sakka and M. Osawa, Hysteresis of Potential-Dependent Changes in Ion Density and Structure of an Ionic Liquid on a Gold Electrode: In Situ Observation by Surface-Enhanced Infrared Absorption Spectroscopy, J. Phys. Chem. Lett., 2013, 4, 3110-3114.

35 S. Baldelli, Surface Structure at the Ionic Liquid-Electrified Metal Interface, Acc. Chem. Res., 2008, 41, 421-431.

36 L. J. Hardwick, J. Saint, I. T. Lucas, M. M. Doeff and R. Kostecki, FTIR and Raman Study of the $\mathrm{Li}_{x} \mathrm{Ti}_{y} \mathrm{Mn}_{1-y} \mathrm{O}_{2}(y=0,0.11)$ Cathodes in Methylpropyl Pyrrolidinium Bis(fluoro-sulfonyl)imide, LiTFSI Electrolyte, J. Electrochem. Soc., 2009, 156, A120. 